\title{
2021 Awards in the Journal of Plant Research
}

\author{
Maki Katsuhara ${ }^{1}$
}

Published online: 8 June 2021

(c) The Botanical Society of Japan 2021

The Botanical Society of Japan honors excellence in publications of the Journal of Plant Research through the Best Paper Awards and the Most-Cited Paper Award every year. We are proud to announce the 2021 recipients.

\section{Best paper awards}

Two papers were selected among regular papers published in 2020.

There are many chloroplast membrane lipid synthases and photosynthesis-related enzymes that originated outside of cyanobacteria. In Sato's paper (2020), a large-scale phylogenetic analysis was conducted and four types of phylogenetic relationships between chloroplast enzymes (encoded by the chloroplast and nuclear genomes) and cyanobacterial counterparts were found: Type 1, chloroplast enzymes diverged from inside of cyanobacterial clade; Type 2, chloroplast and cyanobacterial enzymes are sister groups; Type 3, chloroplast enzymes originated from homologs of bacteria other than cyanobacteria; Type 4, chloroplast enzymes diverged from eukaryotic homologs. These results suggested that phylogenetically diverse gene transfer occurred at different times to establish chloroplasts. Sato (2020) proposes that the host-directed chloroplast formation hypothesis, in which host eukaryotes prepared the membrane lipid synthesis system in advance, should be important for re-examining the origin of chloroplasts. The hypothesis that chloroplasts originated from a single intracellular symbiosis is refuted with a large amount of data presented in this study. This paper is highly evaluated for clarifying the complexity of intracellular symbiosis in the evolution of eukaryotes.

Tokumoto et al. (2020) focused on Polygala paniculata, a non-leguminous Fabales species. About $70 \%$ of all

Maki Katsuhara

jpreic@okayama-u.ac.jp

1 Institute of Plant Science and Resources (IPSR), Okayama

University, 2-10-1, Chuo, Kurashiki 710-0046, Japan angiosperms form symbiotic relationships, arbuscular mycorrhizas (AM), with AM fungi. This symbiosis is estimated to originate over 400 million years ago. On the other hand, root nodule (RN) symbiosis with nitrogen-fixing bacteria, rhizobia and Frankia, has been observed only in limited plants. Recent studies have shown that the origin of RN symbiosis dates back to about 100 million years ago and that it seems to have evolved on the basis of a symbiotic system with AM fungi, but the full story of the evolutionary process of the legume-rhizobia symbiosis is still unclear. In this paper, $P$. paniculata which does not engage in RN symbiosis, was explored to compare with model legumes, such as Lotus japonicus and Medicago truncatula, in order to investigate the evolution of the RN symbiosis system. The phylogenetic position of $P$. paniculata was analyzed and genome size of this species was estimated. The in vitro cultivation conditions and hairy root transformation system were established and optimized. In addition, Tokumoto et al. confirmed P. paniculata can establish symbiosis with AM fungi. Surprisingly, when the plants were infected with the broad host range rhizobium, $P$. paniculata showed a deformation response of the root hairs, which is the initial process of RN symbiosis. This suggests that even the non-RN-producing P. paniculata has a part of the mechanism that initiates RN symbiosis. Thus, this new non-leguminous plant will greatly improve our understanding of how the RN symbiosis system has evolved and been maintained.

\section{Most-cited paper award}

Ronse De Craene's review paper (2018) was published in the special issue "Floral development—Re-evaluation of its importance" published to commemorate the JPR symposium held in 2016. This article outlines that floral evolution is the result of alternating bursts of physical constraints and genetic stabilization processes. The two aspects are related and follow each other in succession. Ronse De Craene concluded that future research needs to combine these different 
perspectives in understanding the evolution of floral systems and their diversification.

Maki Katsuhara

Editor-in-Chief, Journal of Plant Research

\section{References}

Ronse De Craene L (2018) Understanding the role of floral development in the evolution of angiosperm flowers: clarifications from a historical and physico-dynamic perspective. J Plant Res $131: 367-393$

Sato N (2020) Complex origins of chloroplast membranes with photosynthetic machineries: multiple transfers of genes from divergent organisms at different times or a single endosymbiotic event? J Plant Res 133:15-33

Tokumoto Y, Hashimoto K, Soyano T, Aoki S, Iwasaki W, Fukuhara M, Nakagawa T, Saeki K, Yokoyama J, Fujita H, Kawaguchi M (2020) Assessment of Polygala paniculata (Polygalaceae) characteristics for evolutionary studies of legume-rhizobia symbiosis. J Plant Res 133:109-122

Publisher's Note Springer Nature remains neutral with regard to jurisdictional claims in published maps and institutional affiliations. 